\title{
Phylogenetic Structure Analysis Based on Blue Light Receptor Cryptochrome: Insights into How Light Shape the Vertical Structure of Forest Community
}

\author{
Qiming Mei ${ }^{1}$, Ju-Yu Lian ${ }^{1}$, Yue Wang ${ }^{1}$, Meng Li $^{1}$, Xujun Gui ${ }^{1}$, Zheng-Feng Wang ${ }^{1}$, \\ Hong-Lin $\mathrm{Cao}^{1}$, and Wan-Hui $\mathrm{Ye}^{1}$ \\ ${ }^{1}$ South China Botanical Garden, Chinese Academy of Sciences
}

October 13, 2020

\begin{abstract}
Light-regime variability is an important environmental factor which shapes a forest community. So far, none focused on the phylogenetic pattern of plant light receptors, which reflects how genes' evolution influences the coexistence of species in a community. In this study, we analyzed community phylogenetic structure of the south subtropical forest by sequences of plant blue light receptor cryptochrome (CRY) and compared the results of DNA barcodes. Patterns of community assembly was estimated by net relatedness index (NRI) and nearest taxon index (NTI). We found that CRY showed quite different phylogenetic structure as compared to DNA barcoding results, all habitats displayed consistent phylogenetic structure patterns, suggesting a convergent evolution of light sensing system of plant in local adaptation. Also, both NRI and NTI values increased through the time, indicating that the phylogenetic structure of tree community became more overdispersion as succession proceeds; phylogenetic closely-related species tended to co-occur and environmental filtering played a more important role in the community assembly. Furthermore, phylogenetic patterns were more clustering in upper canopy layers, and NTI values of all canopy layers were above zero, suggesting that phylogenetically related species tended to coexist and adapted to similar light conditions.
\end{abstract}

\section{Hosted file}

Manuscript.pdf available at https://authorea.com/users/366771/articles/486413-phylogeneticstructure-analysis-based-on-blue-light-receptor-cryptochrome-insights-into-how-lightshape-the-vertical-structure-of-forest-community 
(a)
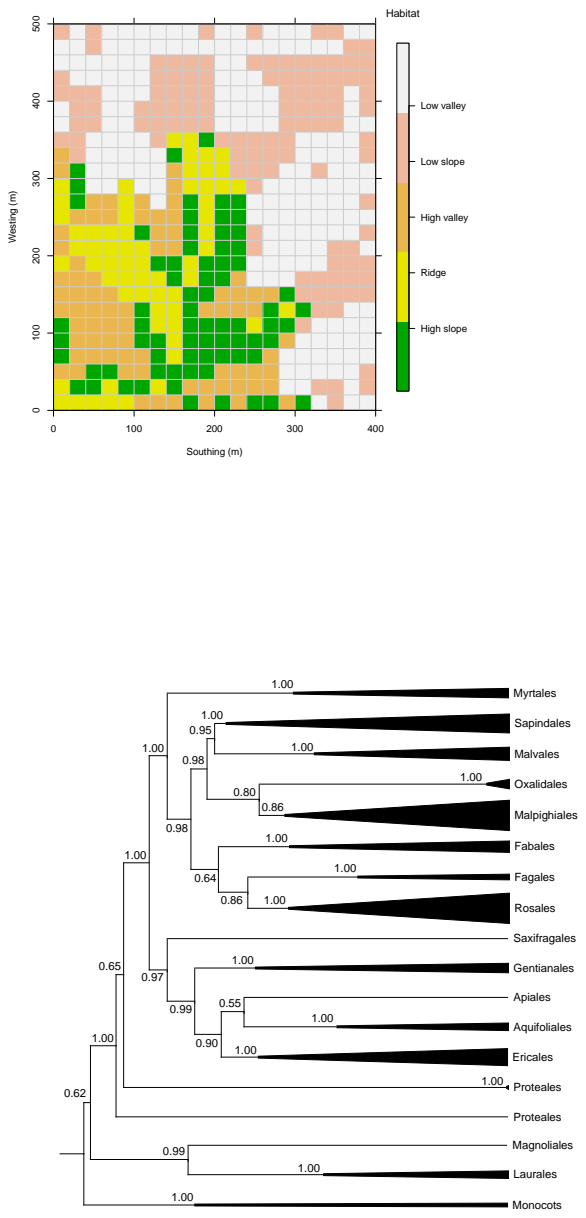

(a) (b)
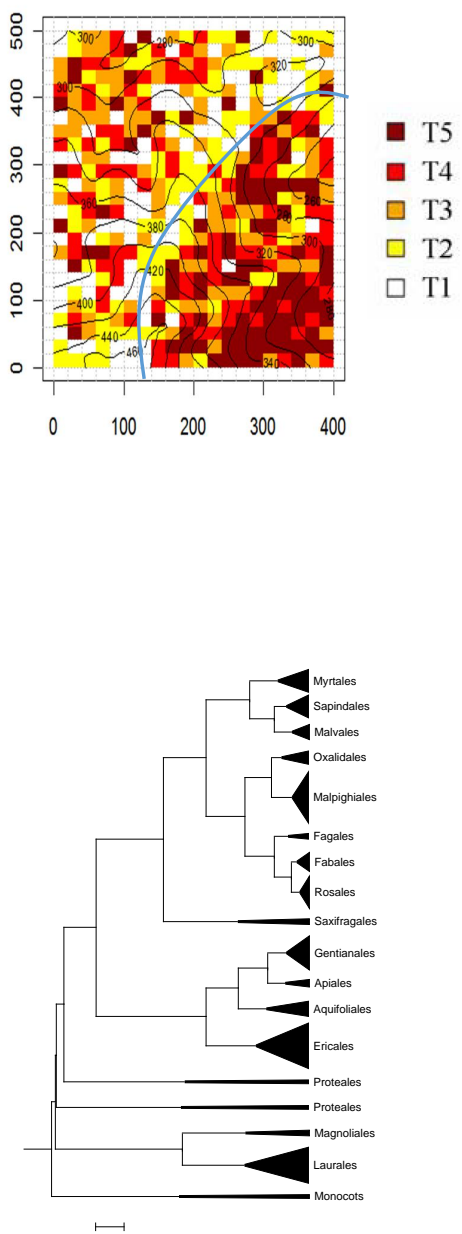

(b) 


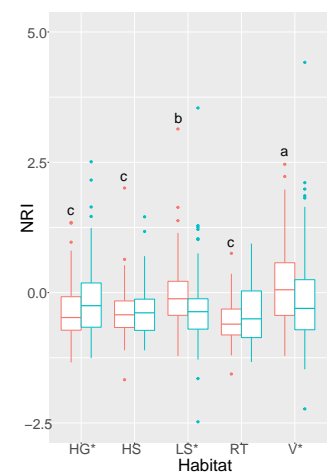

(a)

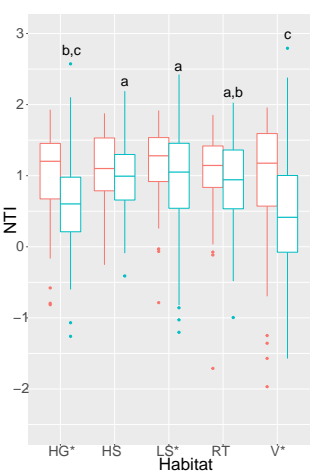

(d)

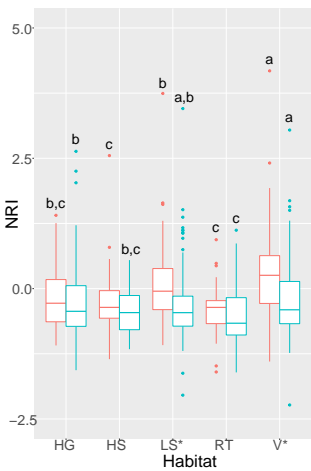

(b)

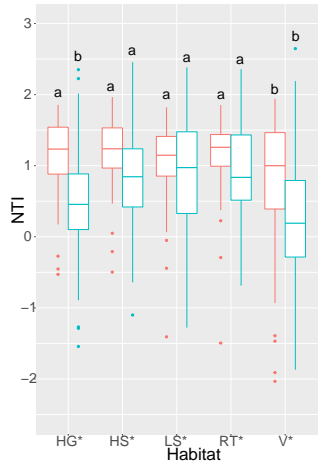

(e)

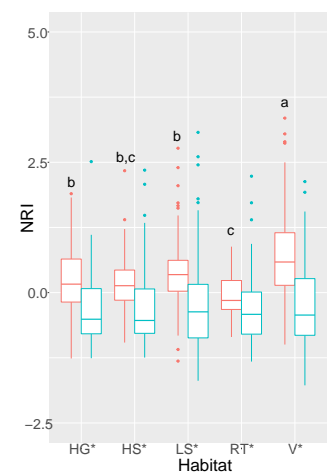

(c)

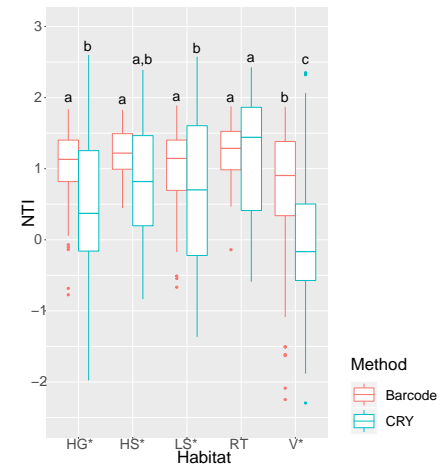

(f)
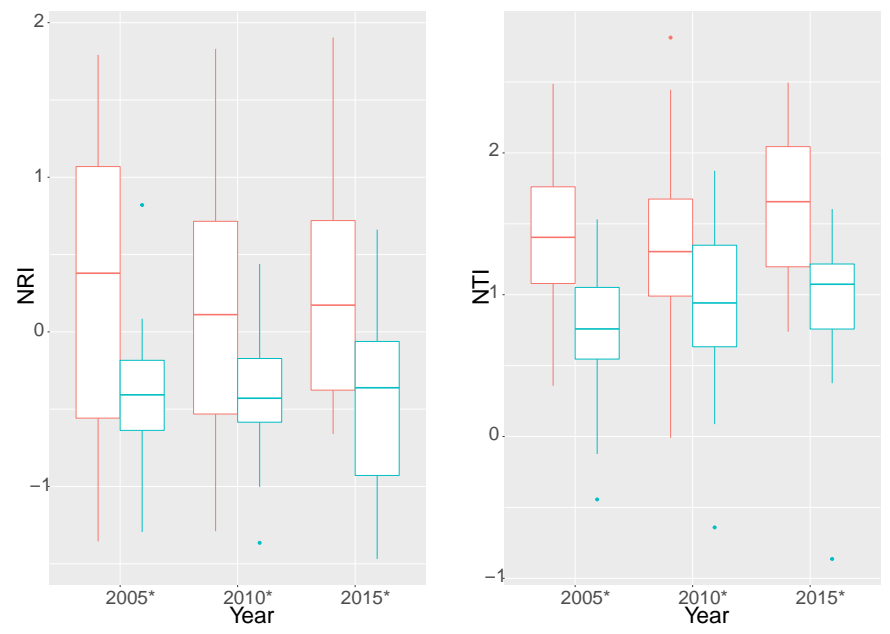

Method

Barcode $\exists \mathrm{CRY}$ 


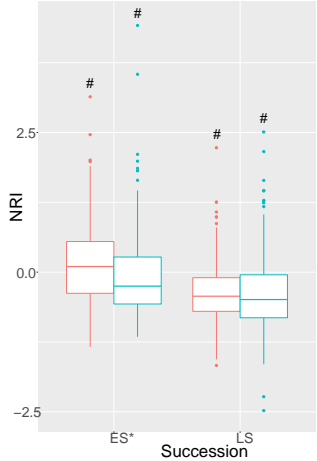

(a)

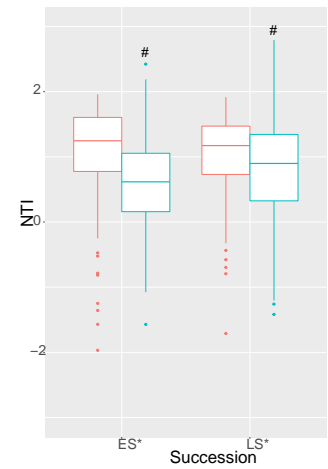

(d)

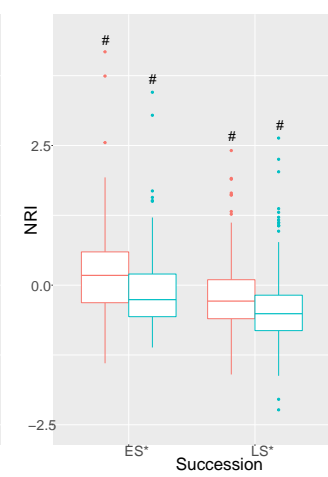

(b)

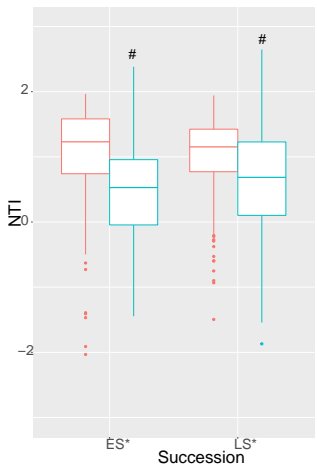

(e)

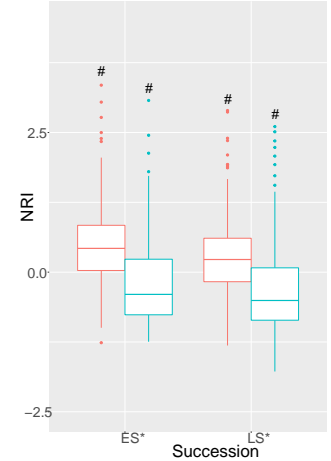

(c)

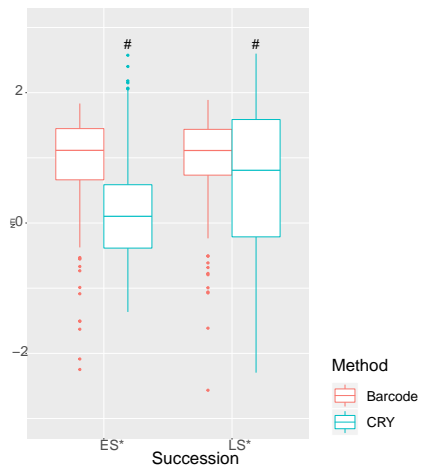

(f) 


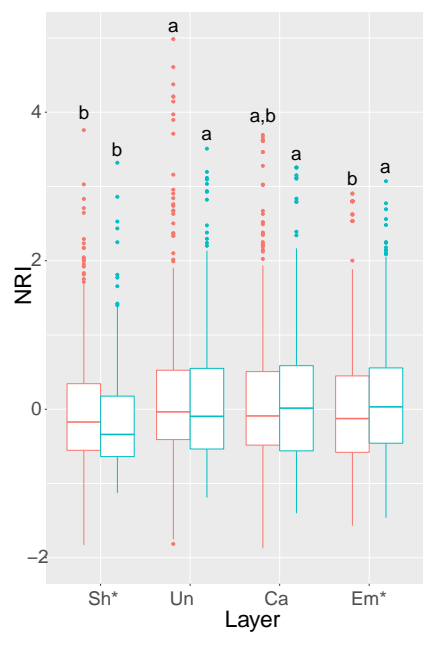

(a)

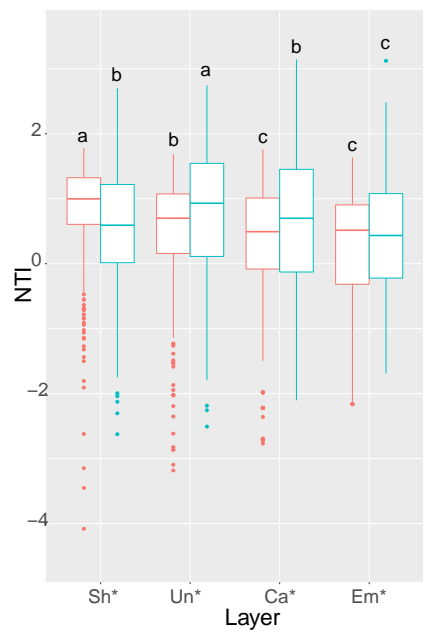

(c)

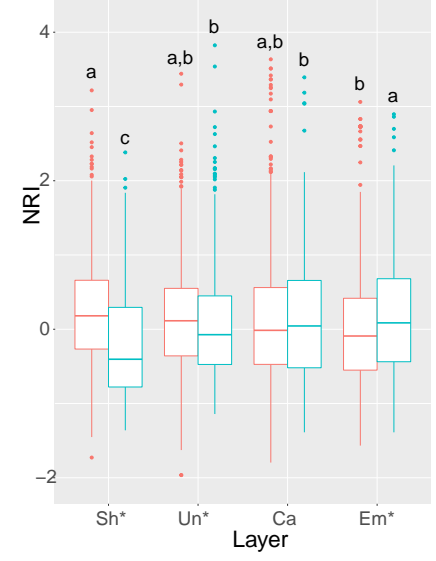

(b)

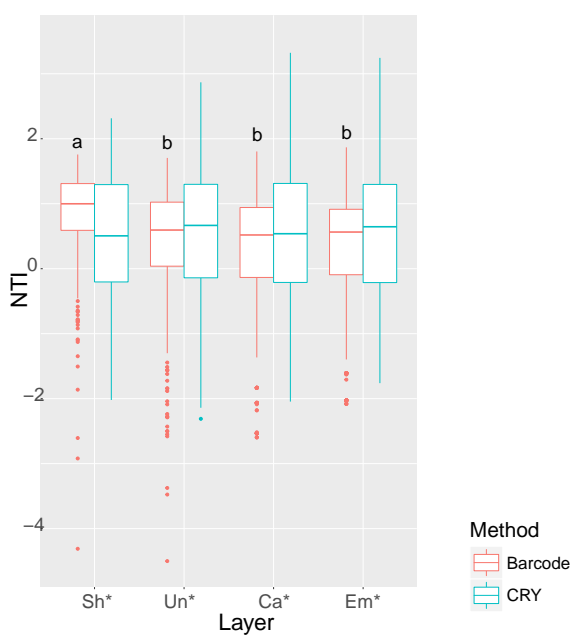

(d) 